\title{
Resource implications of robotic thoracic surgery: what are the wider issues?
}

\author{
Sasha Stamenkovic ${ }^{1}$, Robert D. Slight ${ }^{2}$ \\ ${ }^{1}$ Bart's Health NHS Trust, The Royal London Hospital, Whitechapel, UK; ${ }^{2}$ Freeman Hospital, Newcastle Upon Tyne, UK \\ Correspondence to: Robert D. Slight, PhD. Thoracic Surgeon, Freeman Hospital, Freeman Road, Newcastle Upon Tyne NE7 7DN, UK. \\ Email: bob.slight@nhs.net.
}

The benefits of minimally invasive thoracic surgery are well documented when compared to the use of standard thoracotomy. Much controversy exists, however, regarding the resource implications when using robot-assisted thoracic surgery (RATS), especially when compared to video-assisted thoracoscopic surgery (VATS). Much of the costs attributed to a particular approach center around the frequency and severity of the complications that may arise. Little exists in the literature to appropriately compare and contrast the complication rate following either of the minimally invasive approaches. There is a suggestion that many conventional open surgeons are more readily persuaded to adopt a minimally invasive approach through the use of the robotic platform, therefore reducing the complication-related costs of standard thoracotomy by an increase in minimally invasive resection rates. Further gains may be made in the ability to perform more complex minimally invasive procedures via a RATS approach without recourse to open conversion when compared to VATS. As opportunities and competition increase in the commercial market place, it is reasonable to assume costs will fall and further savings will be made.

Keywords: Robotic; thoracic surgery; cost; outcomes; lobectomy; thymectomy

Submitted Aug 31, 2018. Accepted for publication Nov 22, 2018.

doi: $10.21037 /$ acs.2018.11.10

View this article at: http://dx.doi.org/10.21037/acs.2018.11.10

\section{Resource implications of robotic thoracic surgery, what are the wider issues?}

Robot-assisted thoracic surgery (RATS) has developed over the past 16 years, building on the minimally invasive concepts established by video-assisted thoracoscopic surgery (VATS). Adoption is increasing with the reported benefits including; enhanced vision, greater dexterity, reduced pain and an enhanced learning experience (1-3). The benefits of a minimally invasive approach, whether by RATS or VATS, as compared to open thoracotomy, are well established. These include smaller incisions, less pain, less blood loss, fewer respiratory complications, shortened hospital length of stay (LOS) and a quicker functional return $(4,5)$. Controversy exists, however, regarding the resource implications of a robotic approach, particularly when compared to VATS. This paper will attempt to address some of these issues, while examining some wider considerations.

\section{The costs and complications of VATS}

Complications accrue costs, whether it be due to increased LOS, or the finance involved with additional interventions or treatments. Much of the available literature has therefore focused on the complications associated with both VATS and conventional open surgery (5). Swanson et al. retrospectively examined post-operative complications of both approaches utilizing data from the Society of Thoracic Surgeons database (6). The authors concluded that the combined risk of an adverse event was significantly lower in the VATS group $(\mathrm{P}=0.019)$. A specific, although not statistically significant, finding was that pneumonia was more prevalent in the open group (9.1\% vs. 8.1\%). Cardiac events were found to be significantly more likely following open surgery as was prolonged length of hospital stay.

In a cohort study, Farjah et al. compared the 90-day complication rate between VATS and open surgery, finding 
a statistical difference between the two (30.95\% vs. $53.33 \%$, $\mathrm{P}=0.015$ ) while also documenting a significantly higher readmission rate following open surgery $(\mathrm{P}=0.001)(7)$. A propensity matched analysis by Paul et al. suggested similar findings with a lower morbidity rate following VATS lobectomy (5).

When considering the financial context, perhaps one of the most salient contributions to the VATS $v s$. open debate was made by Casali and Walker (4). They concluded that the operating costs are more expensive for VATS resections, however, this is more than cancelled out by a shorter LOS. Also of note was the financial difference recorded dependent upon the lobe resected by a minimally invasive approach. Unsurprisingly, this was attributable to the number of stapler cartridges used.

\section{The costs and complications of RATS}

The earliest paper analyzing the cost implications of RATS was by Park et al. [2008] (8). Despite the analysis being carried out early on in their learning curve, they concluded that a RATS lobotomy was $\$ 4,000$ cheaper than the corresponding open procedure. Furthermore, it was concluded that greater usage would drive down costs. Much of this saving may relate to quicker recovery times as suggested by Ye et al. when analyzing VATS $v s$. RATS thymectomy. The robotic approach was associated with both a reduced requirement for pleural drainage and shorter LOS $(\mathrm{P}<0.01)(9)$. When considering multiple disciplines, Turchetti et al. concluded that the robotic approach was associated with longer operating times, and therefore the additional cost, than the corresponding laparoscopic procedure, however, this reduced with experience (10).

Again in 2012, Park et al. emphasized the need for a cost-effectiveness study to delineate the monetary costs of RATS in comparison to open thoracotomy and VATS. They also pointed out that such a study assumed equity in clinical efficacy, an assumption that no study has been able to make as no randomized trials have been performed (11). Also touched upon was the fact that the robotic platform may allow for more complex procedures to be performed by a minimally invasive approach than would ordinarily be attempted by VATS. It is clear that as regards the indirect costs of robotic surgery, the more cases that are performed in the lifetime of the machine, the better the cost-efficiency. This is achievable by sharing the use of the robot between several surgeons or different specialty surgeons (11).

\section{The real cost implications of robotic surgery}

In the RATS $v s$. VATS or open cost debate, much attention has focused on the expenditure associated with the system and its disposables. This is a blinkered stance and more consideration has to be given to all factors that consume resources.

\section{Length of stay}

As mentioned above, LOS in most countries is associated with an increase in cost versus a finite fee. A reduction in LOS results in less post-operative hospital expenditure (6). This argument is well rehearsed (7). When considering additional determinants of resources, several factors may be relevant.

\section{Blood loss}

There is very little in the way of blood loss in an uncomplicated robotic operation. In our 5-year experience, the median blood loss is $150 \mathrm{~mL}$, including training cases. We have attributed this to better vision and the use of monopolar and bipolar cautery in each hand (2). There is also more precise picking up and dividing of tissues in robotic surgery which is harder to achieve with a thoracoscopic approach. This aligns with experience in other specialties (12).

\section{Increase in minimally-invasive operations}

The benefits of VATS surgery in terms of hospital stay, pain and functional recovery are well established, all of which may impact favorably on healthcare costs (6). Why, therefore, are more procedures not performed via VATS? One of the principle reasons suggested is the learning curve associated with traditional VATS surgery (13). Some VATS operations may be started but not completed as they are too difficult. Robotic surgery is easier to learn and therefore should result in greater surgeon uptake. In addition, in redo operations, or where the pleural space has been affected by multiple thick inflammatory adhesions, robotic technology comes into its own with excellent vision and enhanced dexterity (2).

More complex pathologies may be addressed. This also applies to sleeve resections for central tumors and thymic surgery which some surgeons consider too difficult or dangerous via a VATS approach (14). With a robotic- 
assisted procedure, and the use of $\mathrm{CO}_{2}$ insufflation to open up a small intra-thoracic space, this becomes more straightforward (15). Operating from the "inside-out", such as in chest wall resections and para-spinal operations, have also become a reality (16). With these more complex operations now possible with the robot platform, the comparison is not between robotic-assisted and VATS, but more appropriately between robotic-assisted and open surgery.

\section{Complications}

In the authors' experience, over a 5-year period there has been a significant reduction in complication rates with the robotic platform as compared to VATS. Visual analogue pain scores have been less (0-1 vs. 4-5) while a 15-fold reduction in neuropraxia rate also being noted. An attendant reduction in chest infection rate was felt to be due to these lower pain scores and an increase in the ability to clear secretions. Similarly, patients with an air leak not requiring suction were able to go home with an ambulatory drainage system in situ, as there were no hospital requirements for analgesia and monitoring.

Farivar et al. analyzed data from the Society of Thoracic Surgeons database and found a significant difference in mortality rates and in the incidence of complications such as prolonged air leak, arrhythmia, atelectasis, pneumonia and re-intubation (17). Adams et al. did not find that roboticassisted surgery was beneficial in comparison to video thoracoscopic surgery in terms of outcomes, but did find that use of the robotic platform resulted in significantly lower postoperative blood transfusion rates $(0.9 \%$ vs. $7.8 \%)$, air leaks for $>5$ days (5.2\% vs. $10.8 \%$ ) and hospital LOS (4.7 vs. 7.3 days) compared with open thoracotomy (18). Kent et al. reported similar findings when comparing robotic to open surgery (19). As compared to VATS, improvements were seen in mortality and complication rates, however, these did not reach statistical significance.

The most recent large-scale paper comparing RATS, VATS and open lobectomy is from Oh et al. who utilized the Premier database for a propensity matched analysis (20). There were statistically significant differences in composite post-operative complications ( $\mathrm{P}=0.0061)$, post-operative bleeding $(\mathrm{P}<0.0001), 30$-day complication rates $(\mathrm{P}=0.0130)$, conversion rates $(\mathrm{P}<0.0001)$ and $\operatorname{LOS}(\mathrm{P}=0.006)$ all in favor of the robotic platform. In addition, patients were more likely to be discharged direct to their home $(\mathrm{P}=0.0108)$ following robotic surgery. The authors suggested the benefits of RATS were attributable to both more surgeons being past their learning curve and technological advances which have allowed more modern incarnations of the robotic platform. Interestingly, they also noted a decrease in open lobectomy (11.5\%), together with a small increase in VATS (1.5\%) and a bigger increase in RATS (10\%), all of which would be in keeping with the aforementioned hypothesis.

Regardless of surgical approach, complications bring an increase in healthcare expenditure. In this regard, Brunelli et al. applied the Ottawa thoracic morbidity and mortality (TM\&M) classification system devised by Seely et al. and linked costs to grade of complication $(21,22)$. The higher grade of complications was directly related to significant increases in the cost of care. A case that had no complications cost $\$ 3,797$, but a case with a $T M \& M$ grade 1 complication cost $\$ 4,908$ and for TM\&M grade IV $\$ 12,590$. In Seely's original retrospective analysis, patients with grade III and IV complications unsurprisingly had greater LOSs than those with grade II $(\mathrm{P}=0.0001)$, coupled with a higher readmission rate $(\mathrm{P}=0.0006)(22)$.

The benefits of minimally invasive surgery as opposed to traditional open surgery in terms of complication rates are well established (17). When considering the RATS $v s$. VATS debate the literature remains limited. Rinieri et al. did attempt to address this question when examining outcomes in patients undergoing both techniques over the course of a year. They noted a significantly higher rate of conversion ( $16 \%$ vs. $9 \%, \mathrm{P}=0.008)$ for VATS patients, a greater amount of segmentectomies performed by robotic techniques, but a non-significant difference between the two with regards to complication rates (23).

\section{What happens next?}

There is no literature to help with this question as most publications focus on learning curve, LOS and cost together with 30- to 90-day mortality outcomes and disease-free survival. Far less attention has been given to functional outcomes and longer-term quality of life. These are factors which may influence an individual's choice of a particular therapeutic intervention and therefore more information is crucial as part of the informed consent process.

Essentially, minimally invasive techniques have been developed as a way of limiting trauma to patients and to allow them to recover more quickly (6). A further key consideration therefore is whether robotic-assisted surgery may increase compliance with adjuvant therapies. 
Shorter recovery times may increase uptake within the current 8 -week window as well as increase compliance and completion rates (24). The societal cost should also be considered, whether this be savings due to a quicker resumption of employment, or caring duties for dependents.

\section{In summary}

The benefits of the robotic approach may include less discomfort than the minimally invasive approach, a higher uptake amongst conventional open surgeons, fewer and less significant complications, shorter hospital stays, quicker return to function and a higher uptake of adjuvant treatments (3). All these factors have a cost implication, but not all a monetary value. As costs decrease with the current commercial opening up of the robot market, our thoughts as clinicians should be concentrated upon how we can make things better for the patient by continuing to evolve our procedures and care as we have always endeavored to do.

\section{Acknowledgements}

None.

\section{Footnote}

Conflicts of Interest: Mr S Stamenkovic is employed as a proctor by Intuitive Surgical. RD Slight has no conflicts of interest to declare.

\section{References}

1. Park BJ, Flores RM, Rusch VW. Robotic assistance for video-assisted thoracic surgical lobectomy: technique and initial results. J Thorac Cardiovasc Surg 2006;131:54-9.

2. Veronesi G. Robotic thoracic surgery: technical considerations and learning curve for pulmonary resection. Thorac Surg Clin 2014;24:135-41, v.

3. Slight RD, Koulaxouzidis G, Stamenkovic S. Sequential robotic-assisted lung resection with a subxiphoid utility incision. Asian Cardiovasc Thorac Ann 2018;26:404-6.

4. Casali G, Walker WS. Video-assisted thoracic surgery lobectomy: can we afford it? Eur J Cardiothorac Surg 2009;35:423-8.

5. Paul S, Altorki NK, Sheng S, et al. Thoracoscopic lobectomy is associated with lower morbidity than open lobectomy: a propensity-matched analysis from the STS database. J Thorac Cardiovasc Surg 2010;139:366-78.
6. Swanson SJ, Meyers BF, Gunnarsson CL, et al. Videoassisted thoracoscopic lobectomy is less costly and morbid than open lobectomy: a retrospective multiinstitutional database analysis. Ann Thorac Surg 2012;93:1027-32.

7. Farjah F, Backhus LM, Varghese TK, et al. Ninetyday costs of video-assisted thoracic surgery versus open lobectomy for lung cancer. Ann Thorac Surg 2014;98:191-6.

8. Park BJ, Flores RM. Cost comparison of robotic, videoassisted thoracic surgery and thoracotomy approaches to pulmonary lobectomy. Thorac Surg Clin 2008;18:297300, vii.

9. Ye B, Tantai JC, Li W, et al. Video-assisted thoracoscopic surgery versus robotic-assisted thoracoscopic surgery in the surgical treatment of Masaoka stage I thymoma. World J Surg Oncol 2013;11:157.

10. Turchetti G, Palla I, Pierotti F, et al. Economic evaluation of da Vinci-assisted robotic surgery: a systematic review. Surg Endosc 2012;26:598-606.

11. Park BJ. Cost concerns for robotic thoracic surgery. Ann Cardiothorac Surg 2012;1:56-8.

12. Farnham SB, Webster TM, Herrell SD, et al. Intraoperative blood loss and transfusion requirements for robotic-assisted radical prostatectomy versus radical retropubic prostatectomy. Urology 2006;67:360-3.

13. Petersen RH, Hansen HJ. Learning curve associated with VATS lobectomy. Ann Cardiothorac Surg 2012;1:47-50.

14. Cerfolio RJ. Robotic sleeve lobectomy: technical details and early results. J Thorac Dis 2016;8:S223-6.

15. Bodner J, Wykypiel H, Wetscher G, et al. First experiences with the da Vinci operating robot in thoracic surgery. Eur J Cardiothorac Surg 2004;25:844-51.

16. Cerfolio RJ, Bryant AS, Minnich DJ. Minimally invasive chest wall resection: sparing the overlying, uninvolved extrathoracic musculature of the chest. Ann Thorac Surg 2012;94:1744-7.

17. Farivar AS, Cerfolio RJ, Vallieres E, et al. Comparing robotic lung resection with thoracotomy and videoassisted thoracoscopic surgery cases entered into the Society of Thoracic Surgeons database. Innovations (Phila) 2014;9:10-5.

18. Adams RD, Bolton WD, Stephenson JE, et al. Initial multicenter community robotic lobectomy experience: comparisons to a national database. Ann Thorac Surg 2014;97:1893-8; discussion 1899-900.

19. Kent M, Wang T, Whyte R, et al. Open, video-assisted thoracic surgery, and robotic lobectomy: review of a national database. Ann Thorac Surg 2014;97:236-42; 
discussion 242-4.

20. Oh DS, Reddy RM, Gorrepati ML, et al. Robotic-Assisted, Video-Assisted Thoracoscopic and Open Lobectomy: Propensity-Matched Analysis of Recent Premier Data. Ann Thorac Surg 2017;104:1733-40.

21. Brunelli A, Drosos P, Dinesh P, et al. The Severity of Complications Is Associated With Postoperative Costs After Lung Resection. Ann Thorac Surg 2017;103:1641-6. 22. Seely AJ, Ivanovic J, Threader J, et al. Systematic

Cite this article as: Stamenkovic S, Slight RD. Resource implications of robotic thoracic surgery: what are the wider issues? Ann Cardiothorac Surg 2019;8(2):250-254. doi: 10.21037/acs.2018.11.10 classification of morbidity and mortality after thoracic surgery. Ann Thorac Surg 2010;90:936-42; discussion 942.

23. Rinieri P, Peillon C, Salaun M, et al. Perioperative outcomes of video- and robot-assisted segmentectomies. Asian Cardiovasc Thorac Ann 2016;24:145-51.

24. D'Amico TA. VATS lobectomy facilitates the delivery of adjuvant docetaxel-carboplatin chemotherapy in patients with non-small cell lung cancer. J Thorac Dis 2016;8:296-7. 